\title{
Should the Diastatic Syndesmosis be Stabilized in Advanced Pronation-External Rotation Ankle Injuries? A Retrospective Cohort Comparison
}

\section{Chi-Chuan Wu ( $\nabla$ ccwu@mail.cgu.edu.tw )}

Chang Gung Memorial Hospital https://orcid.org/0000-0003-3609-7986

\section{Wen-Ling Yeh}

Chang Gung Memorial Hospital Linkou Main Branch: Chang Gung Memorial Hospital

\section{Po-Cheng Lee}

Chang Gung Memorial Hospital Linkou Main Branch: Chang Gung Memorial Hospital

\section{Ying-Chao Chou}

Chang Gung Memorial Hospital Linkou Main Branch: Chang Gung Memorial Hospital

\section{Yung-Heng Hsu}

Chang Gung Memorial Hospital Linkou Main Branch: Chang Gung Memorial Hospital

\section{Yi-Hsun Yu}

Chang Gung Memorial Hospital Linkou Main Branch: Chang Gung Memorial Hospital

\section{Research}

Keywords: Ankle injury, Diastatic syndesmosis, External rotation, Pronation

Posted Date: November 17th, 2020

DOl: https://doi.org/10.21203/rs.3.rs-105420/v1

License: (c) (i) This work is licensed under a Creative Commons Attribution 4.0 International License. Read Full License 


\section{Abstract}

Background: Ankle injuries with the advanced pronation-external rotation (PE) type are relatively uncommon and the debate about whether the diastatic syndesmosis should be stabilized concomitantly has yet achieved a consensus. Comparison of using (Group 1) or non-using (Group 2) screw stabilization for the diastatic syndesmosis was performed retrospectively.

Methods: With the 10-year period, 81 consecutive adult patients with advanced PE ankle injuries (stage 3 or 4 PE type) were treated. After malleolar fractures were internally stabilized with screws and plates, the syndesmotic stability was re-checked by external rotation and hook tests. The necessity of insertion of cortical screws to stabilize diastatic syndesmosis was decided by the individual orthopedic surgeon. The outcomes of both approaches were compared.

Results: Seventy-one patients were followed for at least one year (87.7\%; average, 2 years; range, 1-11 years). Group 1 had 22 patients and Group 2, 49 patients. The union rate in Group 1 was 100\% (22 / 22) and in Group 2, 91.8\% (45 / 49; $p=0.30$ ). Syndesmosis re-diastasis occurred in 13.6\% (3 / 22) of Group 1 and $30.6 \%$ (15 / 49) of Group 2 ( $p=0.13)$. Satisfactory ankle function was noted in $86.4 \%(19 / 22)$ of Group 1 and $65.3 \%$ (32 / 49) of Group 2 ( $p=0.07)$.

Conclusion: Although clinical comparison cannot demonstrate statistical difference, screw stabilization of the diastatic syndesmosis may guarantee safer results. The statistical insignificance may be due to insufficient sample sizes. Clinically and theoretically, insertion of syndesmotic screws to promote ligament healing may be reasonable.

\section{Background}

Ankle injuries are common in the daily activity. Clinically, the majority of ankle injuries are not severe and treatment with various non-surgical techniques can usually achieve a success [1, 2]. However, for some ankle injuries which are normally associated with malleolar fractures and syndesmotic diastasis (SD) often require surgical treatment $[3,4]$. Because the markedly unsymmetrical bony structures (the huge distal tibia and the small lateral malleolus) which are unfavorable for load transfer, ankle injuries are often caused by stresses from various directions [5]. The complex injured modes introduce the difficulties of comprehensive classification. Until now, no any classification is convincingly acknowledged to be applicable for all ankle injuries. Clinically, combined Weber and Lauge-Hansen classifications had been widely used to treat complex ankle injuries by many supporters $[4,6,7]$. They believed that the combined classifications can comprise the majority of common and important ankle injuries.

Pronation-external rotation (PE) injuries by Lauge-Hansen classification are considered often associated with varied degrees of ligamentous injuries $[6,8,9]$. The characteristics of this type of injuries are a medial malleolar fracture or deltoid ligament tear and associated with a spiral fibular fracture several centimeters proximal to the plafond $[6,10]$. In advanced stages of PE injury (stage 3 or 4 ), syndesmotic ligaments and interosseous membrane are believed to be extensively damaged. The syndesmotic 
stability may be lost immediately or progressively. Without effective stabilization for ligament healing, the ankle function will never restore therefore. However, in the literature, some orthopedists still advocate that damage of interosseous membrane is only to a small extent from magnetic resonance imaging (MRI) studies and the damage requires no paying much attention [11].

Clinically, the incidence of PE injuries is evidently lower than supination-external rotation injuries of the ankle $(15 \%$ versus $60 \%)[6,12,13]$. However, it generally requires surgical treatment to restore ankle stability. The most controversial problem is whether the syndesmosis requires to be stabilized concomitantly after internal fixation of associated malleolar fractures $[13,14]$. Although stabilization of the syndesmosis on the spot is simple, removal of the stabilizing screw may require another surgery [15]. Clinically, either approach has been implemented widely but to our knowledge, which is more reasonable has few been studied. The purpose of this retrospective study intended to compare relative superiority of the two approaches in treating advanced PE injuries. Consequently, treatment of this relatively complex injury might become more reasonable.

\section{Methods}

This study had been approved by Institutional Review Board of the authors' institution (IRB: 201900950B0).

\section{Subjects}

From January 1, 2008 to December 31, 2017, 822 consecutive adult patients (> 18 years) who sustained malleolar fractures with or without SD and had been surgically treated were traced for this study. All ankle injuries were classified based on combined Weber and Lauge-Hansen classifications $[4,6,7]$. Ninety-nine cases were grouped as advanced pronation injuries (PE or pronation-abduction injuries) and 81 advanced $P E$ injuries were enrolled in the present study. We defined advanced injuries as the stage of injury being beyond a half of full stages $[6,16]$. In the present study, cases with stages 3 or 4 were considered advanced PE injuries and were enrolled for study. The inclusion criteria for study were all adult patients with stage 3 or 4 PE injuries. The exclusion criteria were association with tibial fractures, old ankle deformities or healed fractures, and congenital or developmental anomalies. The causes of 81 injuries included motorcycle accident (29 cases), slide (16 cases), various sports injuries (10 cases), working injuries ( 6 cases), fall from stairs ( 5 cases), car accident ( 5 cases), and others (10 cases).

At the emergency service or outpatients' department (OPD), patients' general conditions were stabilized first. The clinical and radiological manifestations of the ankle were checked carefully. Anteroposterior and lateral views of the ankle were taken [4]. Computed tomography (CT) scans with three-dimensional (3-D) reconstruction were arranged whenever necessary. The surgery was performed as early as possible (average, 3.5 days; range, $0.5-14$ days).

\section{Surgical procedure}


With the general anesthesia and endotracheal intubation, the patient was placed on the operating table in the supine position. A pneumatic tourniquet was routinely used and an image intensifier was prepared for all surgeries.

The lateral view of contralateral uninjured ankle was taken with the fluoroscope and stored in the picture achieving and communicating system (PACS; GE healthcare, Waukesha, WI, USA) software of our institution immediately [17]. The relative position of the lateral malleolus to the distal tibia was measured [18]. After the concomitant malleolar fractures of injured ankle were openly reduced and internally fixed with screws or plates, the syndesmotic stability was re-checked by external rotational stress and hook tests $[11,19]$. Whether the syndesmosis had to be stabilized with screws depended on surgeon's decision. For cases with screw stabilization, the fibula was temporarily immobilized with a pointed holding clamp on the distal tibia [20]. The relative position of lateral malleolus to the distal tibia was adjusted to match the images of contralateral ankle. One or two $4.5 \mathrm{~mm}$ cortical screws (Synthes, Bettlach, Switzerland) were inserted under image intensifier guidance. After the wound was closed, a short leg splint was applied for all surgical patients.

Postoperatively, the patients were encouraged to ambulate with protected weight bearing as early as possible. They were followed at the OPD with the 4-6 weeks interval.

Clinical and radiological healing processes were recorded. The splint was discontinued to use at 6 weeks. The syndesmotic screw was removed based on the surgeon's policy. The removal of internal fixation for malleolar fractures required beyond one year.

The fracture was considered union as clinically, no pain or tenderness locally; radiographically, the fracture gap being vanished or connected with solid callus [21]. The nonunion was defined as the fracture was yet united beyond 9 months.

The ankle function was assessed with America Orthopedic Foot and Ankle Society (AOFAS) score [22]. The total scores were 100 points and included three categories (pain, function, and alignment). An excellent result was no less than 90 points and a good result, no less than 80 points. A satisfactory outcome comprises an excellent or good result.

\section{Statistical analysis}

A SPSS version 20 (SPSS Inc., Chicago, IL, USA) software was used for statistical comparison. $\mathrm{P}<0.05$ was considered statistically significant. The chi-square test was used for categorical data and the Mann-Whitney $U$ test was used for numerical data.

\section{Results}

Seventy-one patients were followed for at lease one year (average, 2.0 years; range, 1-11 years). Ten patients were lost despite all possible efforts to contact them. The follow-up rate was (87.7\%, 71 / 81). 
The 71 patients were divided into two groups: Group 1 (22 patients with syndesmotic screw stabilization) and Group 2 (49 patients without syndesmotic screw stabilization). The follow-up rate of Group 1 was $84.6 \%$ (22 / 26) and Group 2, 89.1\% (49 / 55).

The Group 1 included 17 men and 5 women (average, 36 years; range, 18-77 years). The syndesmotic screws were removed in 20 patients (average, 2.5 months; range, 1.5-5 months). The Group 2 included 23 men and 26 women (average, 42 years; range, 18-78 years).

Fracture union was achieved in all 22 patients in the Group 1 (100\% of union rate; Table 1). Two patients in the Group 2 had medial malleolar nonunions and two other patients had fibular nonunions ( $91.8 \%$ of union rate, $p=0.30$ ).

One deep wound infection occurred in Group 1 patients and two, in Group 2 patients. All were recovered after once debridement and local care $(p=0.93)$.

One syndesmotic screw broke at 6 months and it occurred in one of the two cases without early removal of the screw.

Syndesmotic diastasis recurred in 13.6\% (3 / 22) of Group 1 patients and 30.6\% (15 / 49) of Group 2 patients $(p=0.13$; post-hoc power $=0.31$; Figs. 1,2$)$. Syndesmotic re-diastasis was defined as the tibiofibular clear space further separated more than $2 \mathrm{~mm}$ based on immediately postoperative and the latest comparison of anteroposterior radiographs of the ankle $[19,21]$.

Ankle osteoarthritis occurred in four Group 2 patients $(8.6 \%, 4 / 49)$ and none in Group 1 patients ( $p=$ 0.58; Fig. 3).

Ankle function was assessed by AOFAS scores. The Group 1 had an average score of 82 points (range, 74-88 points) and Group 2, an average of 80 points (range, $66-86$ points; $p=0.06$ ).

A satisfactory ankle function occurred in 86.4\% (19 / 22) of Group 1 patients and 65.3\% (32 / 49) of Group 2 patients $(p=0.07$; post-hoc power $=0.44$; Figs. 4,5$)$.

\section{Discussion}

In normal human gait, when one foot touches the ground, the center of body gravity will move to the contralateral side and introduces bending and rotational moments [23]. Because the marked size disparity between the distal tibia and the lateral malleolus, the ankle contour becomes very unsymmetrical. Not only is the load transfer unsmooth, but also is the movement of talus greatly restricted [24]. Once stresses from various directions are subjected to the ankle, various complex injuries will occur. Clinically, among various ankle injuries requiring treatment, supination-external rotation injuries are reported most common (around $60 \%$ ) and PE injuries, around 15\%. However, the severity of the latter is much higher and generally requires surgical intervention $[6,10,12-14]$. 
In advanced PE injuries (stage 3 or 4), the stability of medial aspect of tibia is lost first because of medial malleolar fractures or deltoid ligament tear. Consequently, the anterior inferior tibiofibular ligament tear follows. Then, the fibular shaft several centimeters proximal to the plafond (commonly $>6 \mathrm{~cm}$ ) fractures spirally and interosseous membrane is disrupted from the syndesmosis upwards to the fractured fibula. This is the standard patho-mechanism of stage $3 \mathrm{PE}$ injuries. If the damage continuously extends, the posterior malleolus will fracture or posterior inferior tibiofibular ligament is disrupted. So far, a stage $4 \mathrm{PE}$ injury is developed $[6,13,14,25]$. In the literature, either injury is regarded as frankly or potentially unstable and surgical treatment is normally necessary $[7,8]$.

Surgical techniques for treatment of advanced PE injuries are controversial. Some orthopedic surgeons declare that once the medial malleolar fracture is stabilized with screws and the fibular fracture is immobilized with a splint with or without plate fixation, the syndesmotic stability may be sufficient. Therefore, syndesmotic screw stabilization may be unnecessary $[10,26]$. However, much more orthopedic surgeons object this concept. They believe that extensive destruction of ligaments and interosseous membrane is a potential risk for syndesmotic re-diastasis after walking. The talus will continuously push the lateral malleolus posterolaterally. A 1.5 months of splint immobilization is insufficient for healing of ligaments and interosseous membrane $[8,11]$. Despite lack of statistical significance in the present study, the ratio of re-diastasis (30.6\% versus $13.6 \%$ ) may remind of potential danger of ignorance. The statistical insignificance may be due to insufficient sample sizes (post-hoc power $=0.31$ ). Re-diastasis of syndesmosis had been reported to cause progressive osteoarthritis in the literature $[1,8,11,27,28]$.

In the stance phase of gait, the talus will be pushed with $3^{0}-5^{0}$ of external rotation and $1.5 \mathrm{~mm}$ of lateral shift [29]. Consequently, the anteromedial aspect of lateral malleolus will bear intermittent stresses towards posterolateral direction. A non-stabilized syndesmosis should be very difficult to withstand this load when all surrounding ligaments and interosseous membrane are without resistant function. Theoretically, a stabilizing screw should be inserted $[8,30]$.

The outcomes of syndesmotic re-diastasis may require long-term observation. It is dissimilar to an acute $\mathrm{SD}$, which is always combined with malleolar fractures. Patients are generally unable to weight bearing [31]. However, syndesmotic re-diastasis is the only remaining sequela and the disasters will deteriorate insidiously. It may therefore cause the ankle function without statistical difference ( $86.4 \%$ versus $65.2 \%$ of satisfactory rate, $p=0.07$; post-hoc power $=0.44$ ) between the two groups in the present study. Increased sample sizes and longer follow-up may reflect the true clinical features.

The vascularity of syndesmosis has been enthusiastically studied. The perforating branch of peroneal artery mainly supplies the surrounding ligaments [24,32]. In advanced PE injuries, the interosseous membrane tears from the upper edge of the syndesmosis upwards to the fractured fibula $[6,13,14,26$, 33]. The blood supply is very possible to be severely compromised and hinders the ligament healing. Immobilization with a short leg splint for simply six weeks runs a high risk of re-diastasis. In the present study, $30.6 \%$ of the re-diastatic rate is found. Insertion of a stabilizing screw lowers the rate to $13.6 \%$ ( $p=$ $0.31)$. 
Syndesmotic stabilization may be via a screw insertion or suture-button technique [31, 34]. Although the latter is gradually supported by some surgeons recently, the former still has merits of technical simplicity and a high success rate as long as screw removal is delayed beyond 3 months $[7,15]$.

Some limitations may exist in the present study. (1) Sample sizes are insufficient and follow-up periods are not long enough (average of 2 years). Therefore, although the ratios of re-diastasis and satisfactory ankle function are evidently different between the two groups, statistical comparison is still insignificant. After all, advanced PE injuries are not so common that enlarging case numbers may require much longer time. Clinically, comparison via a cohort study may be less possible. The sample sizes required for study of re-diastasis are 92 of each group, and for satisfactory ankle function, 63 of each group in the present study. (2) The optimal time for removal of syndesmotic screw or without removal cannot be guaranteed. Screw breakage or re-diastasis may let surgeons hesitate to insert a stabilizing screw. Recently, without removal of syndesmotic screws seem to achieve more supports $[3,15,35]$.

\section{Conclusion}

Although clinical comparison cannot demonstrate statistical difference, screw stabilization of diastatic syndesmosis may guarantee safer results. The statistical insignificance may be due to insufficient sample sizes. Clinically and theoretically, insertion of syndesmotic screws to promote ligament healing may be reasonable.

\section{Abbreviations}

AOFAS: American Orthopedic Foot and Ankle Society; CT: computed tomography; MRI: magnetic resonance imaging; OPD: outpatients' department; PACS: picture achieving and communicating system; PE: pronation-external rotation; SD: syndesmotic diastasis; SPSS: Statistical Product and Service Solutions; 3-D: three-dimensional.

\section{Declarations}

\section{Acknowledgements}

Not applicable.

\section{Author's contributions}

CCW was responsible for study design, data analysis, and writing manuscript. Other five authors were responsible for providing and analyzing data.

\section{Funding}

This study did not receive any funding support. 
Availability of data and materials

All data were available with submission.

Ethics approval and consent to participate

This study had been approved by Institutional Review Board of the authors' institution

(IRB: 201900950B0). Consent to participate was unnecessary.

\section{Consent for publication}

Not applicable.

\section{Competing interests}

The authors do not have any conflict of interests relevant to this study.

\section{Author details}

All authors are working at the Department of Orthopedic Surgery, Chang Gung Memorial

Hospital, Chang Gung University, Taoyuan City, Taiwan.

\section{References}

1. Verhage SM, Schipper IB, Hoogendoorn JM. Long-term functional and radiographic outcomes in 243 operated ankle fractures. J Foot Ankle Res. 2015;8:45.

2. McKeown R, Rabiu AR, Ellard DR, Kearney RS. Primary outcome measures used in interventional trials for ankle fractures: A systematic review. BMC Musculoskelet Disord. 2019;20:388.

3. Michelson JD, Wright M, Blankstein M. Syndesmotic ankle fractures. J Orthop Trauma. 2018;32:104.

4. Al-Hourani K, Stoddart M, Chesser TJS. Syndesmotic fixation in unstable ankle fractures: Does early post-operative weight bearing affect radiographic outcomes? Injury 2019; 50: 790-795.

5. Gougoulias N, Sakellariou A. When is a simple fracture of the lateral malleolus not so simple? how to assess stability, which ones to fix and the role of the deltoid ligament. Bone Joint J. 2017;99-B:8515.

6. Okanobo H, Khurana B, Sheehan S, Duran-Mendicuti A, Arianjam A, Ledbetter S. Simplified diagnostic algorithm for Lauge-Hansen classification of ankle injuries. Radiographics. 2012;32:E71-84.

7. D’Hooghe P, York PJ, Kaux JF, Hunt KJ. Fixation techniques in lower extremity syndesmotic injuries. Foot Ankle Int. 2017;38:1278-88.

8. Nousiainen MT, McConnell AJ, Zdero R, McKee MD, Bhandari M, Schemitsch EH. The influence of the number of cortices of screw purchase and ankle position in Weber $\mathrm{C}$ ankle fracture fixation. $\mathrm{J}$ Orthop 
Trauma. 2008;22:473-8.

9. Chen Y, Qiang M, Zhang K, Li H, Dai H. A reliable radiographic measurement for evaluation of normal distal tibiofibular syndesmosis: a multiple-detector computed tomography study in adults. J Foot Ankle Res. 2015;8:32.

10. Hinds RM, Schottel PC, Berkes MB, Little MT, Helfet DL, Lorich DG. Evaluation of Lauge-Hansen designation of Weber C fractures. J Foot Ankle Surg. 2014;53:434-9.

11. Park YH, Yoon MA, Choi WS, Choi GW, Hong SJ, Kim HJ. The predictive value of MRI in the syndesmotic instability of ankle fracture. Skeletal Radiol. 2018;47:533-40.

12. Robertson GA, Wood AM, Aitken SA, Court Brown C. Epidemiology, management, and outcome of sport-related ankle fractures in a standard UK population. Foot Ankle Int. 2014;35:1143-52.

13. Schottel PC, Berkes MB, Little MT, Garner MR, Fabricant PD, Lazaro LE, et al. Comparison of clinical outcome of pronation external rotation versus supination external rotation ankle fractures. Foot Ankle Int. 2014;35:353-9.

14. Warner SJ, Schottel PC, Hinds RM, Helfet DL, Lorich DG. Fracture-dislocation demonstrate poorer postoperative functional outcomes among pronation external rotation IV ankle fractures. Foot Ankle Int. 2015;36:641-7.

15. Dingemans SA, Rammelt S, White TO, Goslings JC, Schepers T. Should syndesmotic screws be removed after surgical fixation of unstable ankle fractures? a systematic review. Bone Joint J. 2015;98-B:1497-504.

16. van den Heuvel SB, Dingemans SA, Gardenbroek TJ, Schepers T. Assessing quality of syndesmotic reduction in surgically treated acute syndesmotic injuries: A systematic review. J Foot Ankle Surg. 2019;58:144-50.

17. Wu CC. Is clinical measurement of anatomic axis of the femur adequate? Acta Orthop. 2017;88:40710.

18. Grenier S, Benoit B, Rouleau DM, Leduc S, Laflamme GY, Liew A. APTF: anteroposterior tibiofibular ratio, a new reliable measure to assess syndesmotic reduction. J Orthop Trauma. 2013;27:207-11.

19. Pakarinen H, Flinkkila T, Ohtonen P, Hyvonen P, Lakovaara M, Leppilahti J, et al. Intraoperative assessment of the stability of the distal tibiofibular joint in supination-external rotation injuries of the ankle: sensitivity, specificity, and reliability of two clinical tests. J Bone Joint Surg Am. 2011;93:2057-61.

20. Phisitkul P, Ebinger T, Goetz J, Vaseenon T, Marsh JL. Forceps reduction of the syndesmosis in rotational ankle fractures: a cadaveric study. J Bone Joint Surg Am. 2012;94:2256-61.

21. Hsu YT, Wu CC, Lee WC, Fan KF, Tseng IC, Lee PC. Surgical treatment of syndesmotic diastasis: emphasis on effect of syndesmotic screw on ankle function. Int Orthop. 2011;35:359-64.

22. Kitaoka HB, Alexander IJ, Adelaar RS, Nunley JA, Myerson MS, Sanders M. Clinical rating systems for the ankle-hindfoot, midfoot, hallux, and lesser toes. Foot Ankle Int. 1994;15:349-53. 
23. Wu CC, Shih $\mathrm{CH}$. Biomechanical analysis of the dynamic hip screw in the treatment of intertrochanteric fractures. Arch Orthop Trauma Surg. 1991;110:307-10.

24. Akoh CC, Phisitkul P. Anatomic ligament repairs of syndesmotic injuries. Orthop Clin North Am. 2019;50:401-14.

25. Blom RP, Meijer DT, de Muinck Keizer RO, Stufkens SAS, Sierevelt IN, Schepers T, et al. Posterior malleolar fracture morphology determines outcome in rotational type ankle fractures. Injury. 2019;50:1392-7.

26. Hermans JJ, Beumer A, de Long TA, Kleinrensink GJ. Anatomy of the distal tibiofibular syndesmosis in adults: a pictorial essay with a multimodality approach. J Anatomy. 2010;217:633-45.

27. Segal G, Elbaz A, Parsi A, Heller Z, Palmanovich E, Nyska M, et al. Clinical outcomes following ankle fracture: a cross-sectional observational study. J Foot Ankle Res. 2014;7(1):50.

28. Warner SJ, Fabricant PD, Garner MR, Schottel PC, Helfet DL, Lorich DG. The measurement and clinical importance of syndesmotic reduction after operative fixation of rotational ankle fractures. $J$ Bone Joint Surg Am. 2015;97:1935-44.

29. Sasse M, Nigg BM, Stefanyshyn DJ. Tibiotalar motion- effect of fibular displacement and deltoid ligament transection: in vivo study. Foot Ankle Int. 1999;20:733-7.

30. Schepers T. To retain or remove the syndesmotic screw: A review of literature. Arch Orthop Trauma Surg. 2011;131:879-83.

31. Van Heest TJ, Lafferty PM. Injuries to the ankle syndesmosis. J Bone Joint Surg Am. 2014;96:60313.

32. Penera K, Manji K, Wedel M, Shofler D, Labovitz J. Ankle syndesmotic fixation using two screws: risk of injury to the perforating branch of the peroneal artery. J Foot Ankle Surg. 2014;53:534-8.

33. Warner SJ, Garner MR, Hinds RM, Helfet DL, Lorich DG. Correlation between the Lauge-Hansen classification and ligament injuries in ankle fractures. J Orthop Trauma. 2015;29:574-8.

34. McKenzie AC, Hesselholt KE, Larsen MS, Schmal H. A systematic review and meta-analysis on treatment of ankle fractures with syndesmotic rupture: Suture-button fixation versus cortical screw fixation. J Foot Ankle Surg. 2019;58:946-53.

35. Dingemans SA, Birnie MFN, Sanders FRK, van den Bekerom MPJ, Backes M, van Beeck E, et al. Routine versus on demand removal of the syndesmotic screw: a protocol for an international randomized controlled trial (RODEO-trial). BMC Musculoskelet Disord. 2018;19:35.

\section{Tables}

Table 1 Comparison between with or without using syndesmotic screws for advanced pronation-external rotation ankle injuries $(n=71)$ 


\begin{tabular}{|llll|}
\hline Items & With & Without & p value \\
\hline $\mathbf{n}(\mathbf{n = 2 2})$ & $\mathbf{( n = 4 9 )}$ & & \\
\hline Union rate & $100 \%$ & $91.80 \%$ & 0.3 \\
\hline Wound infection & $4.5 \%(1 / 22)$ & $4.1 \%(2 / 49)$ & 0.93 \\
\hline Re-diastasis & $13.6 \%(3 / 22)$ & $30.6 \%(15 / 49)$ & 0.13 \\
\hline Osteoarthritis & 0 & $8.6 \%(4 / 49)$ & 0.58 \\
\hline AOFAS score (points) & 82 & 80 & 0.06 \\
\hline Satisfactory rate & $86.4 \%(19 / 22)$ & $65.3 \%(32 / 49)$ & 0.07 \\
\hline AOFAS: American Orthopedic Foot and Ankle Society. & \\
\hline
\end{tabular}

\section{Figures}

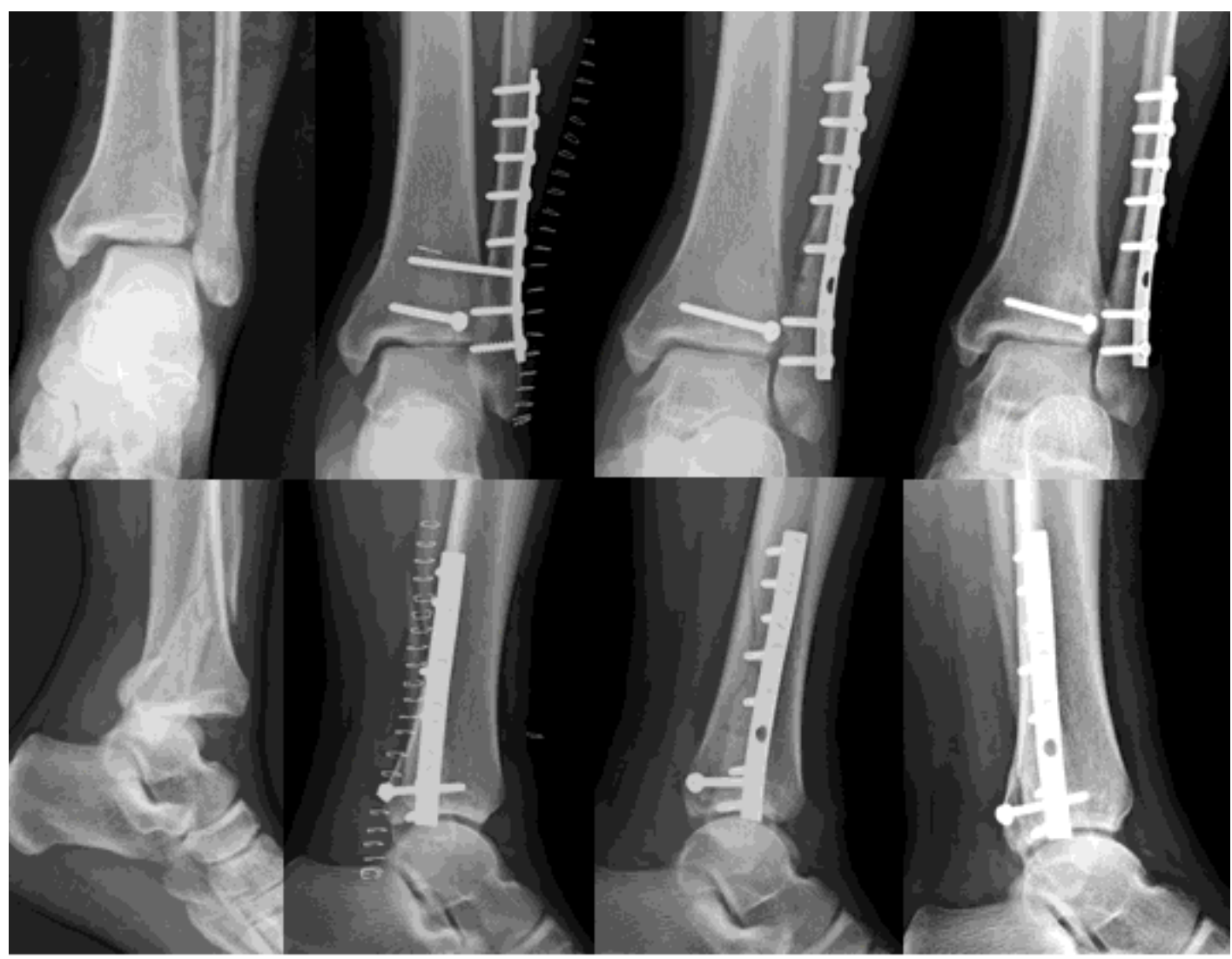

Figure 1

A 52-year-old man sustained a left stage 4 pronation-external rotation ankle injury due to fall from stairs. Bimalleolar fractures were treated with screws and plates. Diastatic syndesmosis was stabilized with a cortical screw. The syndesmotic screw was removed at 1.5 months. Re-diastasis occurred and the patient had a fair ankle function at 6-year 


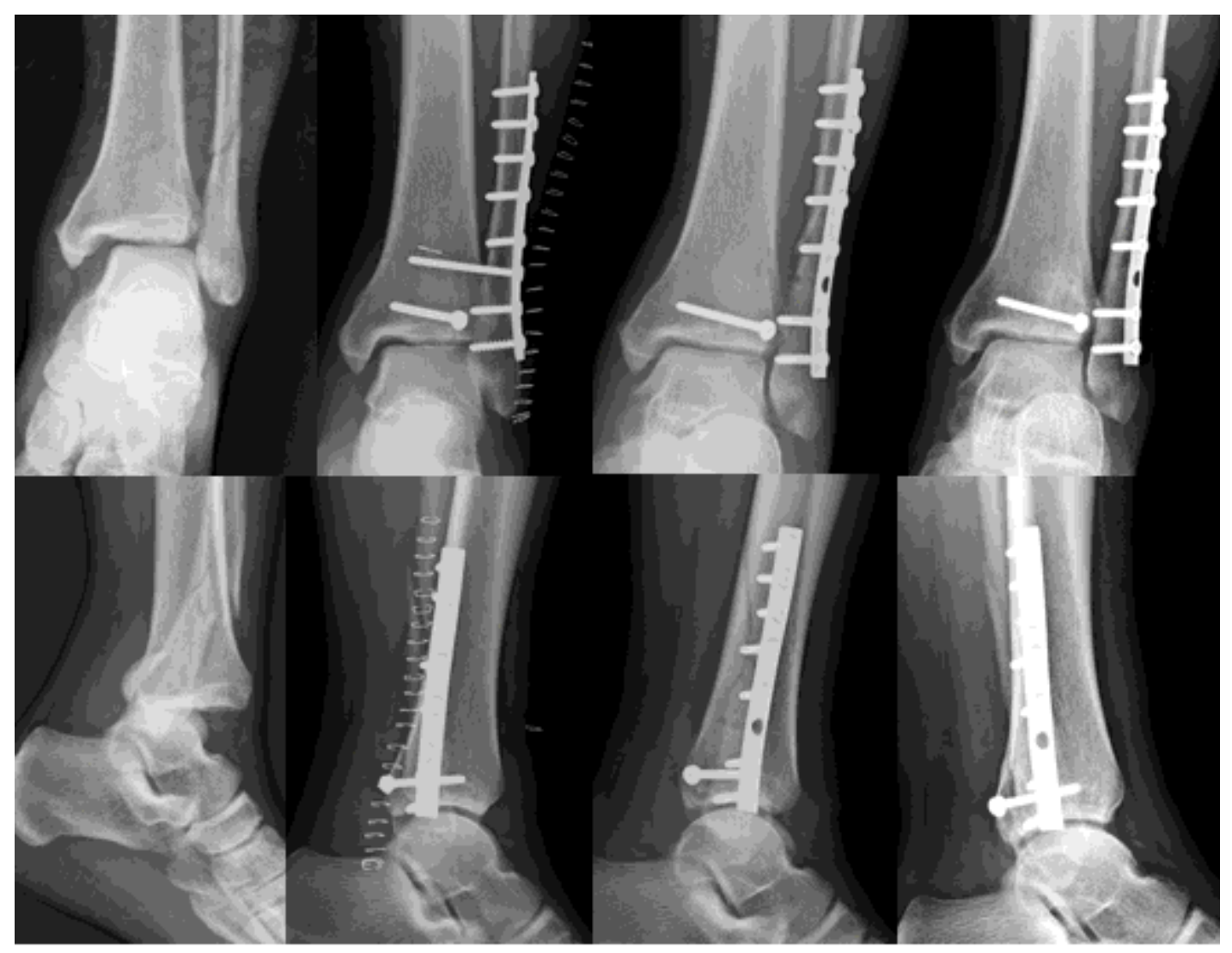

\section{Figure 1}

A 52-year-old man sustained a left stage 4 pronation-external rotation ankle injury due to fall from stairs. Bimalleolar fractures were treated with screws and plates. Diastatic syndesmosis was stabilized with a cortical screw. The syndesmotic screw was removed at 1.5 months. Re-diastasis occurred and the patient had a fair ankle function at 6-year 


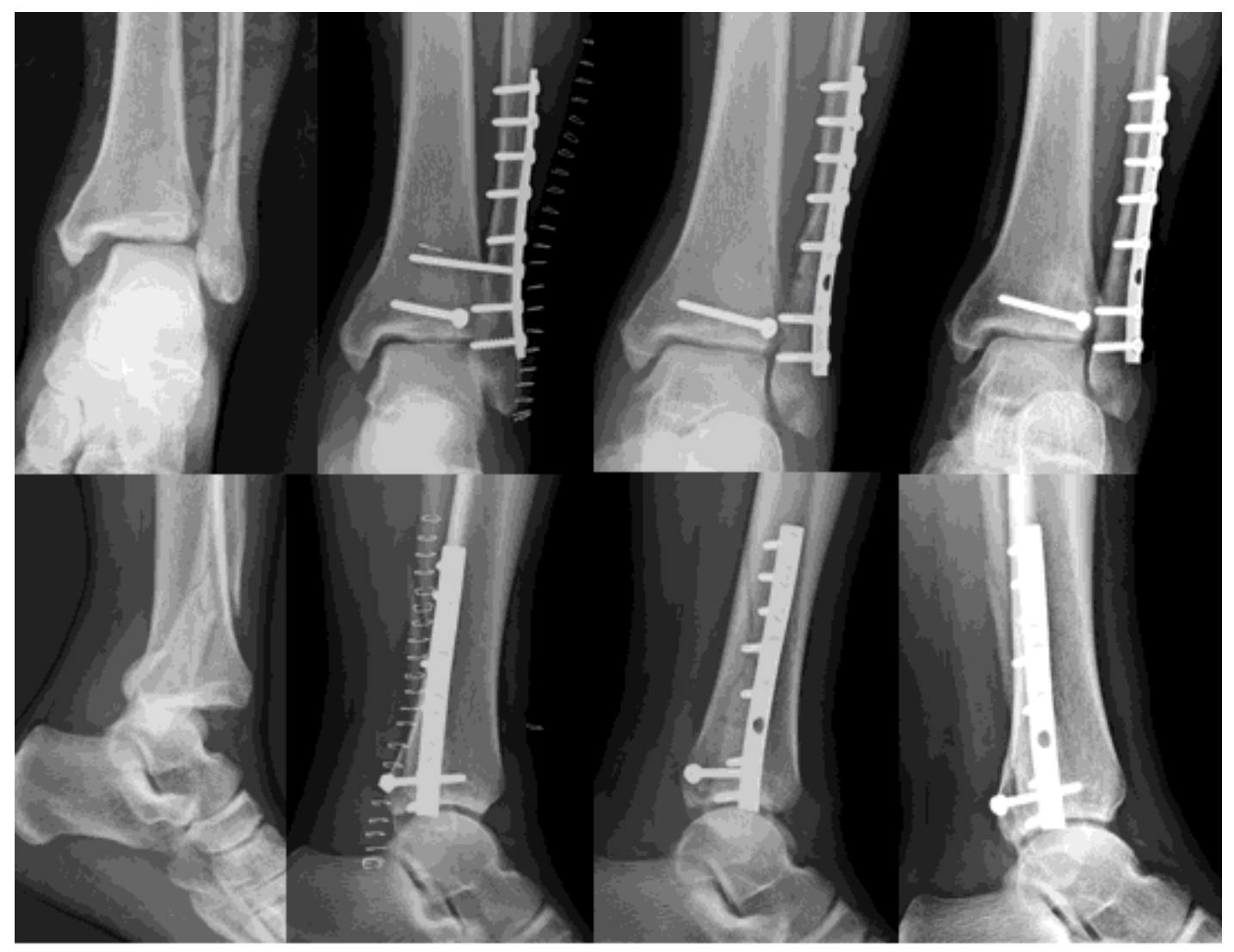

\section{Figure 1}

A 52-year-old man sustained a left stage 4 pronation-external rotation ankle injury due to fall from stairs. Bimalleolar fractures were treated with screws and plates. Diastatic syndesmosis was stabilized with a cortical screw. The syndesmotic screw was removed at 1.5 months. Re-diastasis occurred and the patient had a fair ankle function at 6-year 


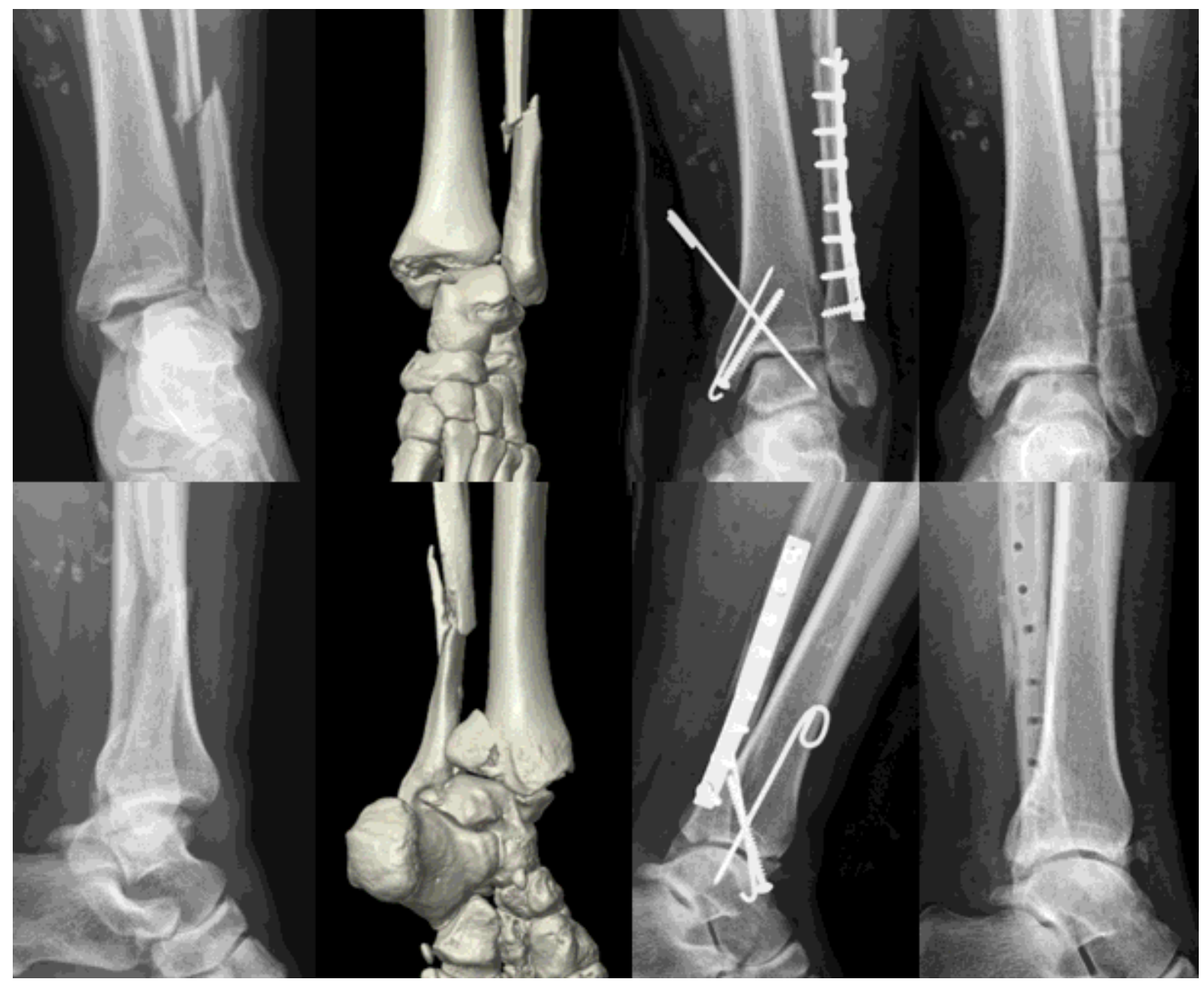

Figure 2

A 42-year-old woman sustained a left stage 4 pronation-external rotation ankle injury due to slide. Reconstructive computed tomography (CT) scans confirmed the lesions. Bimalleolar fractures were treated with screws, Kirschner wires, and plates. Diastatic syndesmosis was not stabilized. Re-diastasis with lateral shift of the talus occurred and the patient had a fair ankle function at 2.5-year follow-up. 


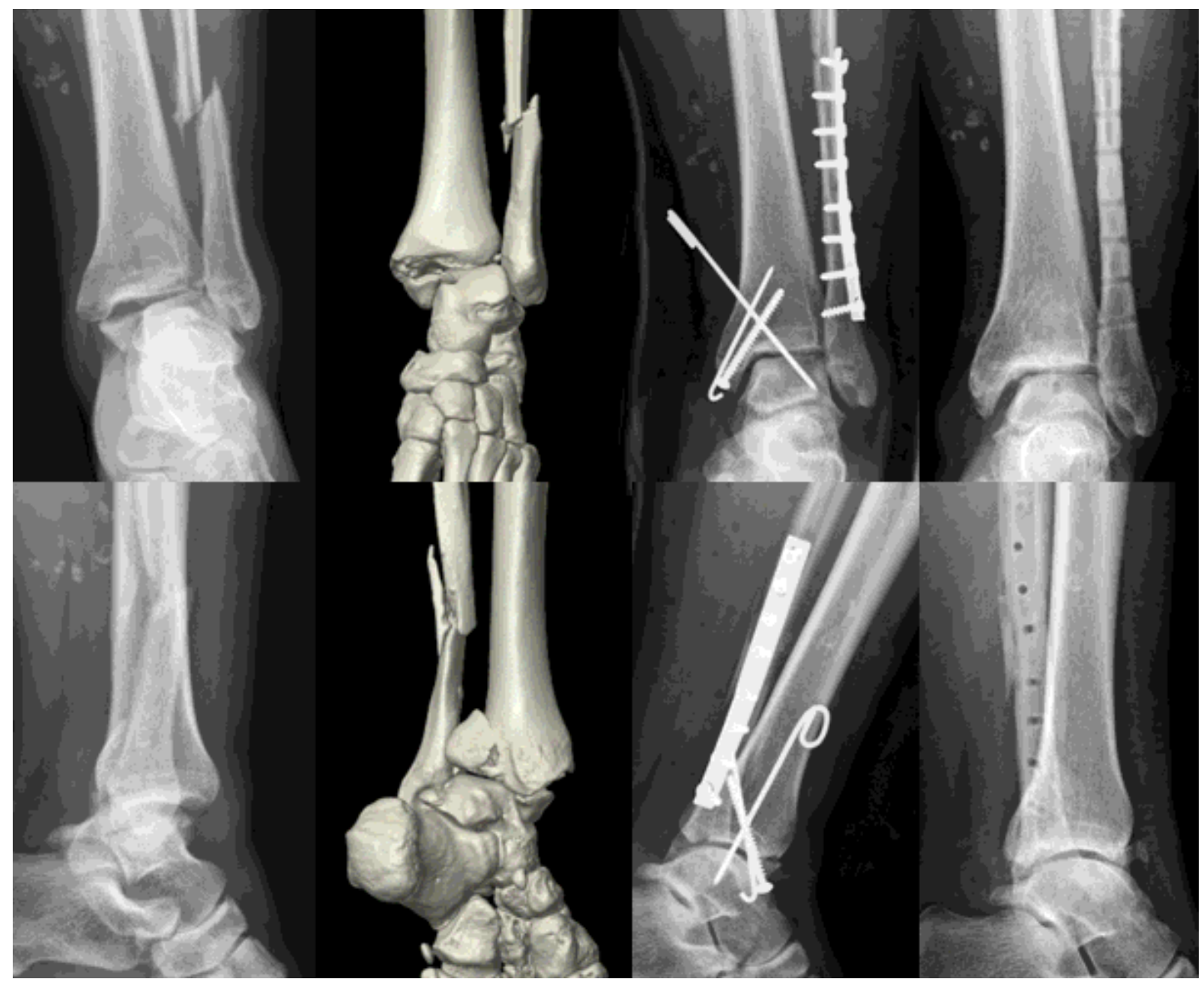

Figure 2

A 42-year-old woman sustained a left stage 4 pronation-external rotation ankle injury due to slide. Reconstructive computed tomography (CT) scans confirmed the lesions. Bimalleolar fractures were treated with screws, Kirschner wires, and plates. Diastatic syndesmosis was not stabilized. Re-diastasis with lateral shift of the talus occurred and the patient had a fair ankle function at 2.5-year follow-up. 


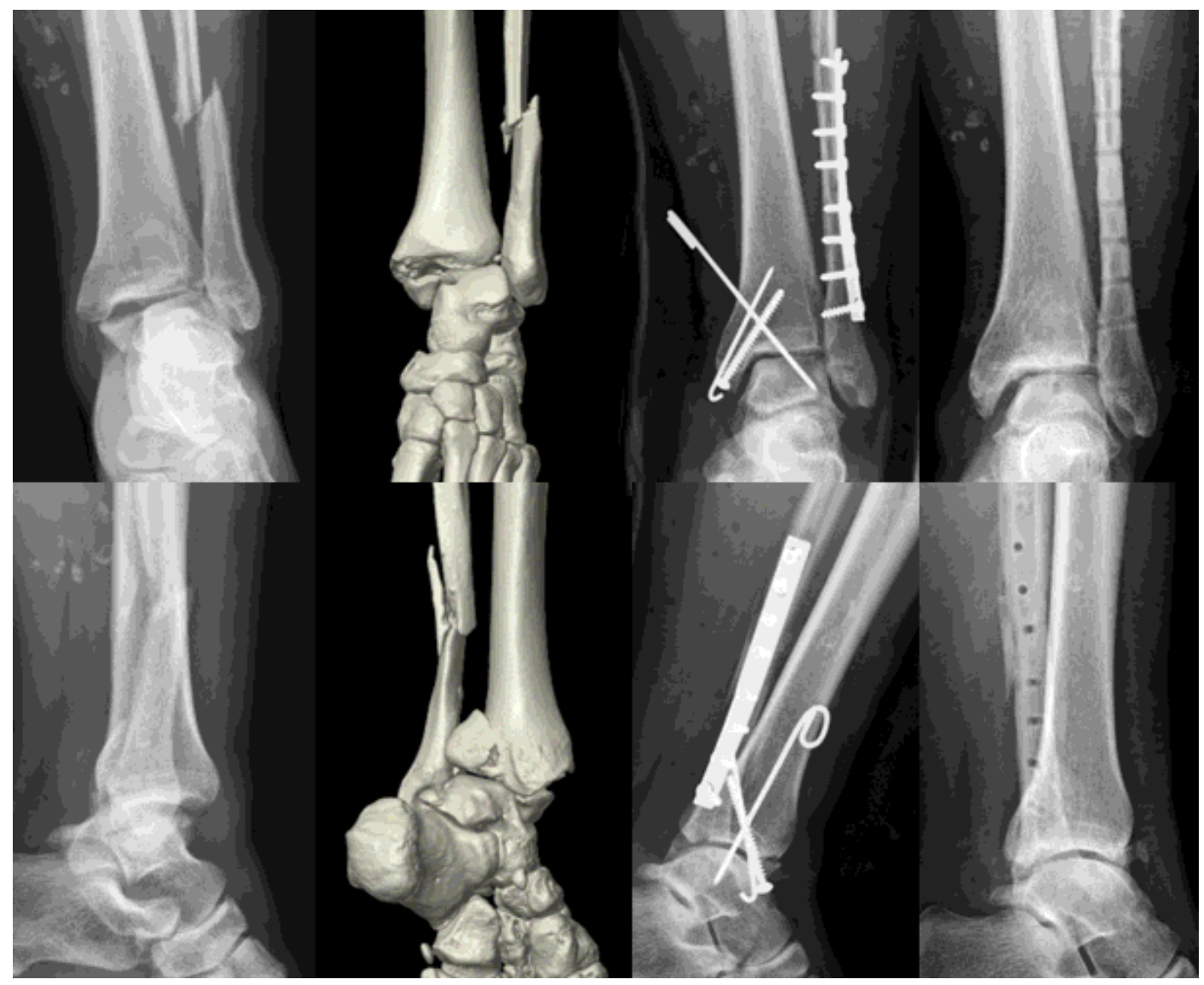

Figure 2

A 42-year-old woman sustained a left stage 4 pronation-external rotation ankle injury due to slide. Reconstructive computed tomography (CT) scans confirmed the lesions. Bimalleolar fractures were treated with screws, Kirschner wires, and plates. Diastatic syndesmosis was not stabilized. Re-diastasis with lateral shift of the talus occurred and the patient had a fair ankle function at 2.5-year follow-up. 


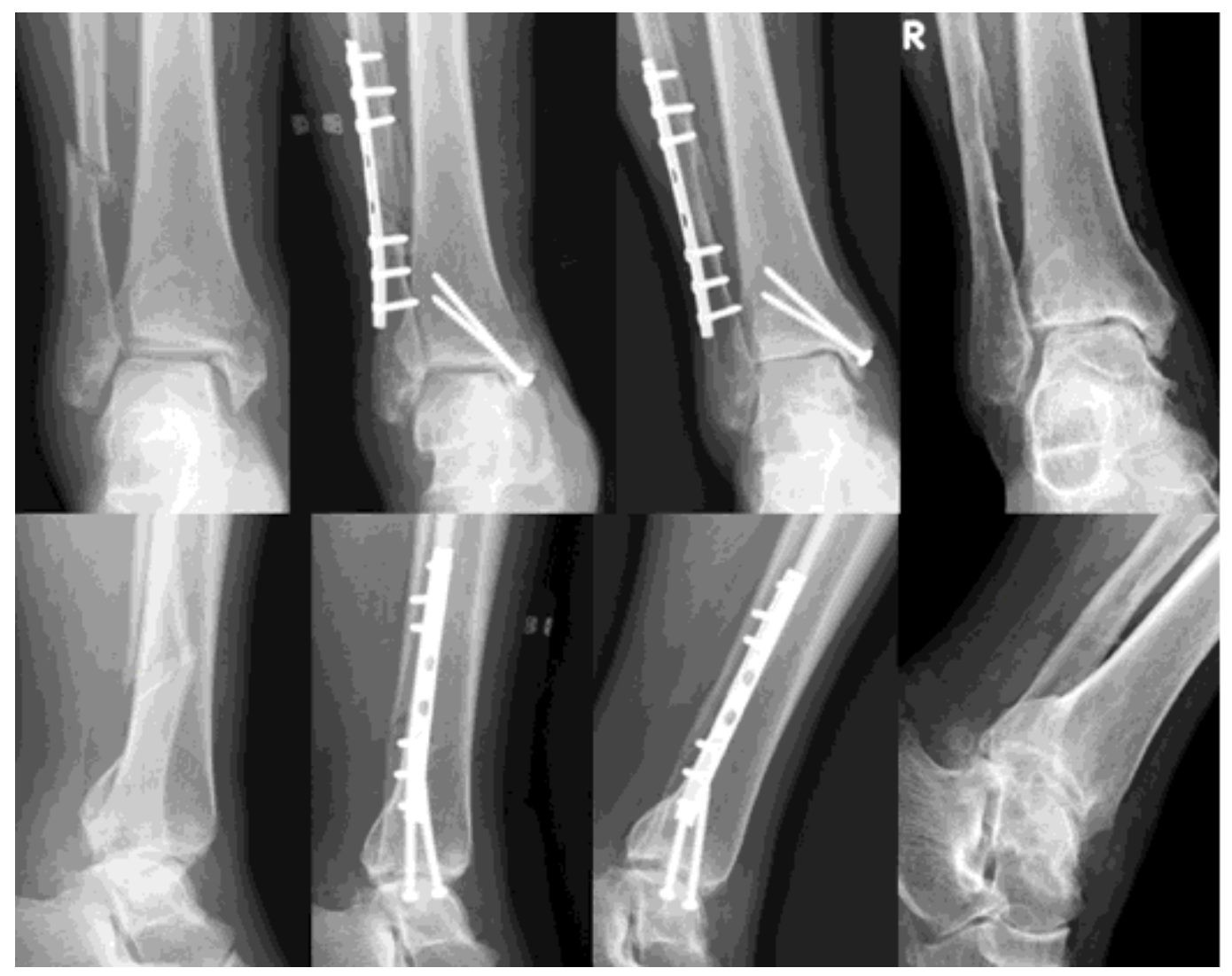

Figure 3

A 60-year-old woman sustained a right stage 4 pronation-external rotation ankle injury due to motorcycle accident. Bimalleolar fractures were treated with screws and plates. Diastatic syndesmosis was not stabilized. The malleolar implants were removed at 1.5 years. Re-diastasis with ankle osteoarthritis occurred and the ankle function was poor at 11-year follow-up. 


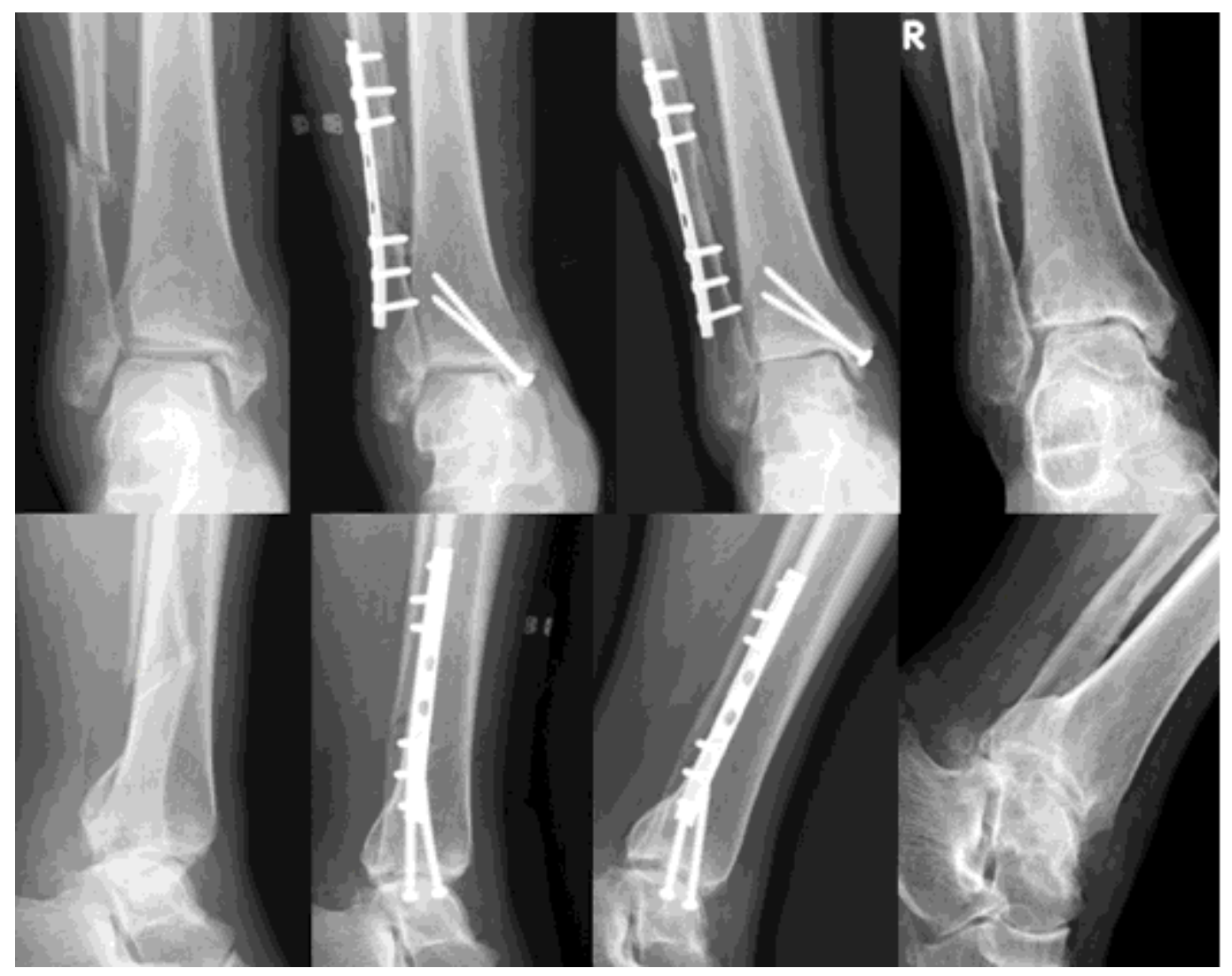

Figure 3

A 60-year-old woman sustained a right stage 4 pronation-external rotation ankle injury due to motorcycle accident. Bimalleolar fractures were treated with screws and plates. Diastatic syndesmosis was not stabilized. The malleolar implants were removed at 1.5 years. Re-diastasis with ankle osteoarthritis occurred and the ankle function was poor at 11-year follow-up. 


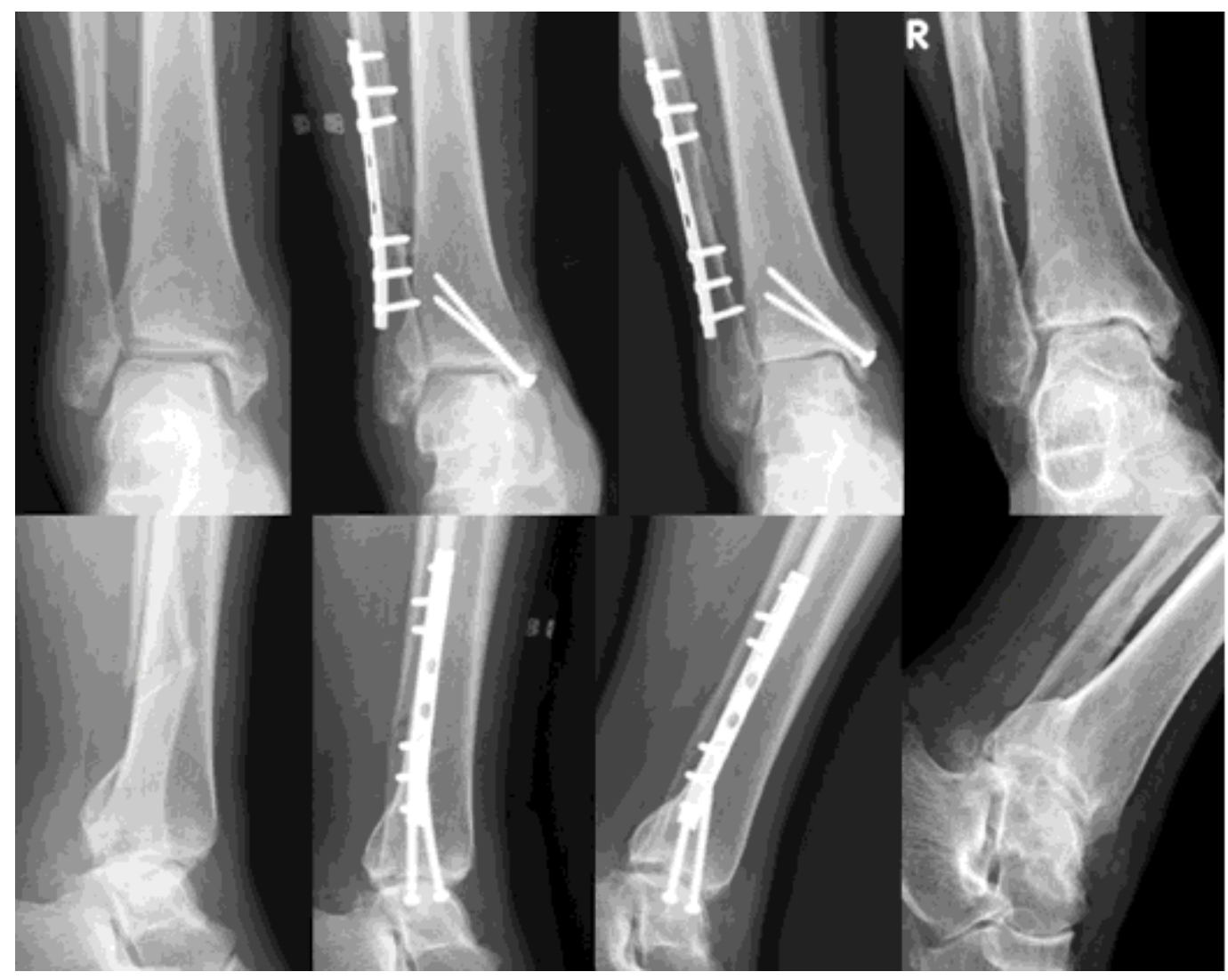

Figure 3

A 60-year-old woman sustained a right stage 4 pronation-external rotation ankle injury due to motorcycle accident. Bimalleolar fractures were treated with screws and plates. Diastatic syndesmosis was not stabilized. The malleolar implants were removed at 1.5 years. Re-diastasis with ankle osteoarthritis occurred and the ankle function was poor at 11-year follow-up. 


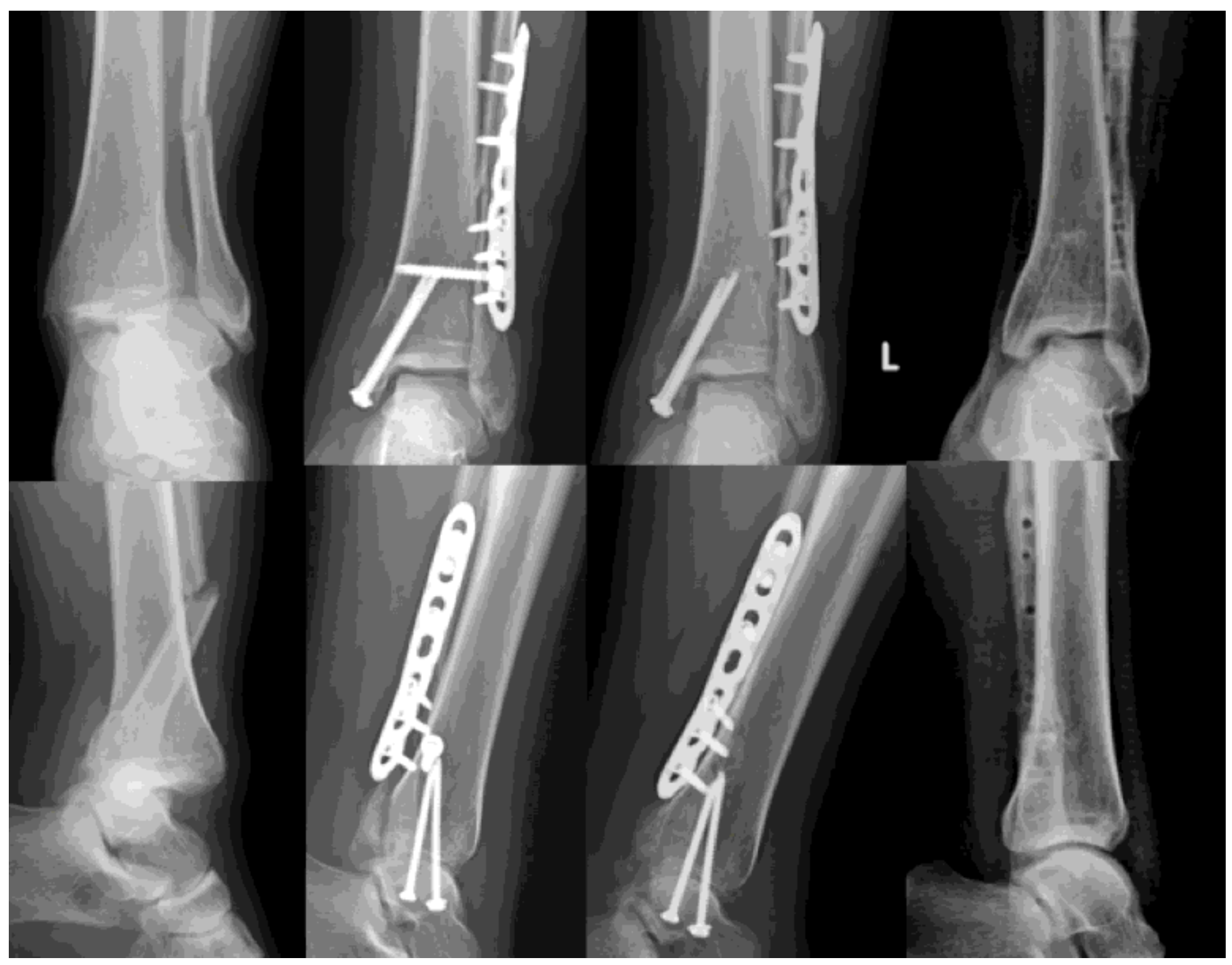

\section{Figure 4}

A 26-year-old man sustained a left stage 4 pronation-external rotation ankle injury due to motorcycle accident. Bimalleolar fractures were treated with screws and plates. Diastatic syndesmosis was stabilized with a cortical screw. The syndesmotic screw was removed at 4 months. The patient had a good ankle function at 2.4-year follow-up. 


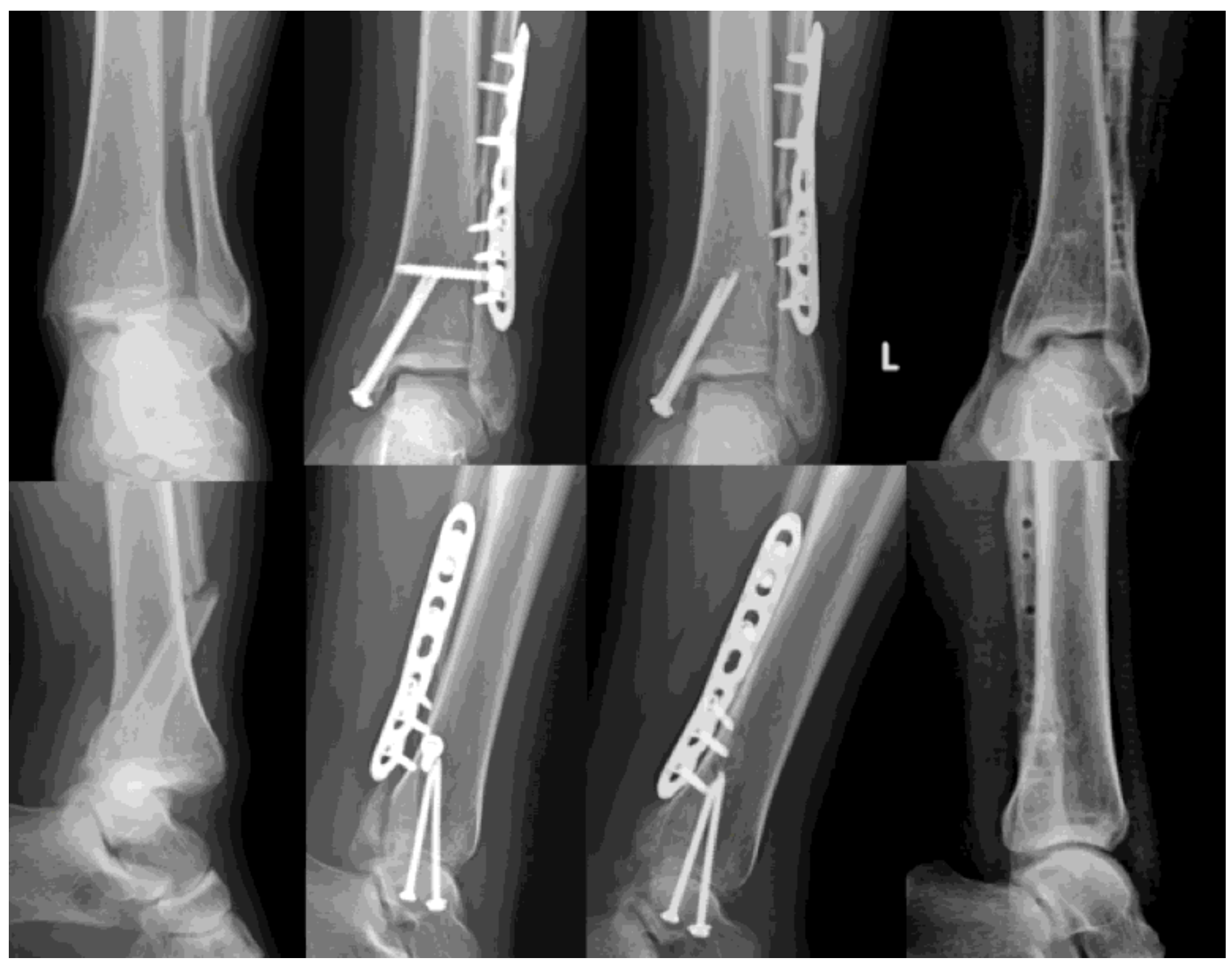

\section{Figure 4}

A 26-year-old man sustained a left stage 4 pronation-external rotation ankle injury due to motorcycle accident. Bimalleolar fractures were treated with screws and plates. Diastatic syndesmosis was stabilized with a cortical screw. The syndesmotic screw was removed at 4 months. The patient had a good ankle function at 2.4-year follow-up. 


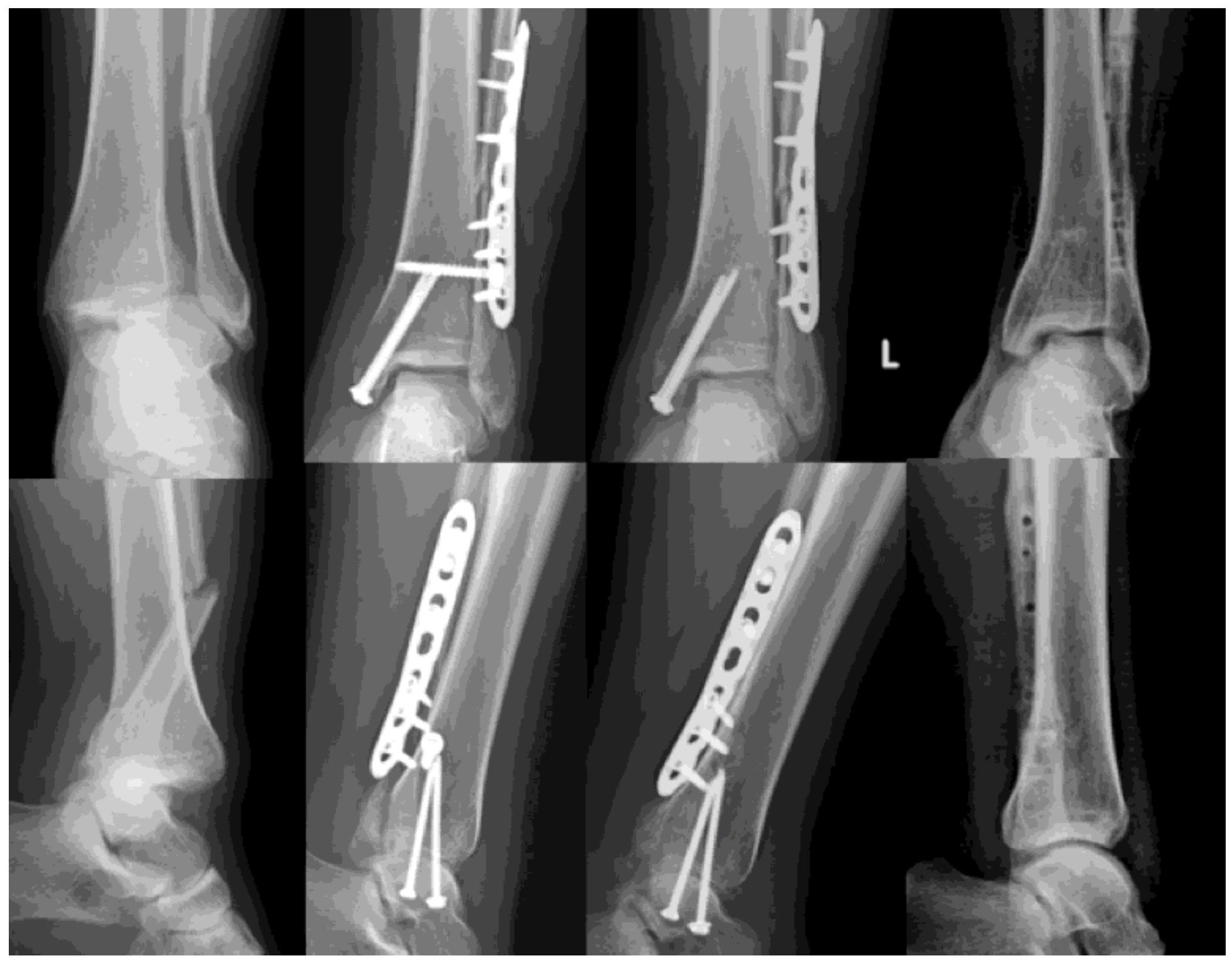

\section{Figure 4}

A 26-year-old man sustained a left stage 4 pronation-external rotation ankle injury due to motorcycle accident. Bimalleolar fractures were treated with screws and plates. Diastatic syndesmosis was stabilized with a cortical screw. The syndesmotic screw was removed at 4 months. The patient had a good ankle function at 2.4-year follow-up. 


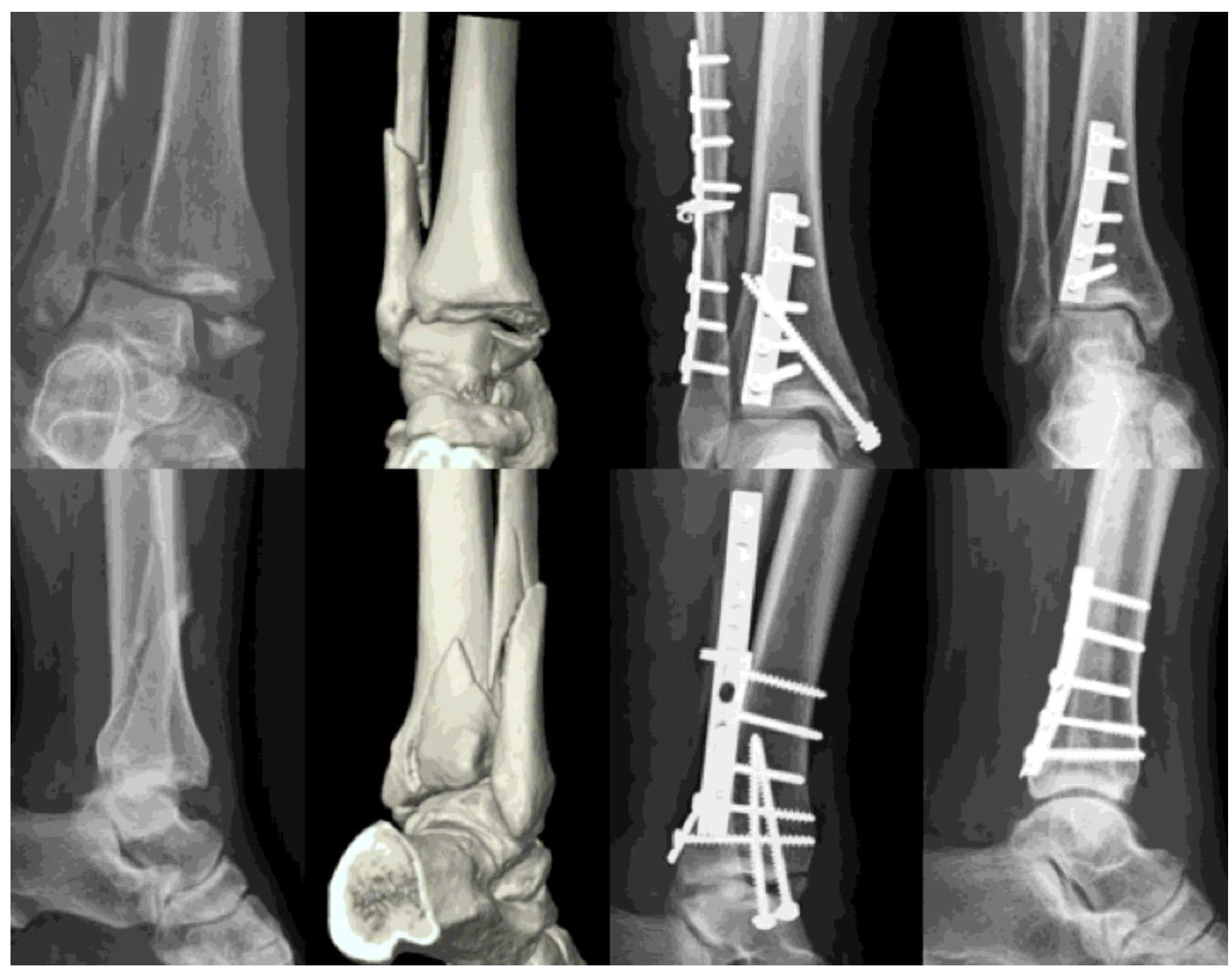

Figure 5

A 56-year-old woman sustained a right stage 4 pronation-external rotation ankle injury due to slide. Reconstructive computed tomography (CT) scans confirmed the lesions. Trimalleolar fractures were treated with screws and plates. Diastatic syndesmosis was not stabilized. The patient had a good ankle function at 4.3-year follow-up. 


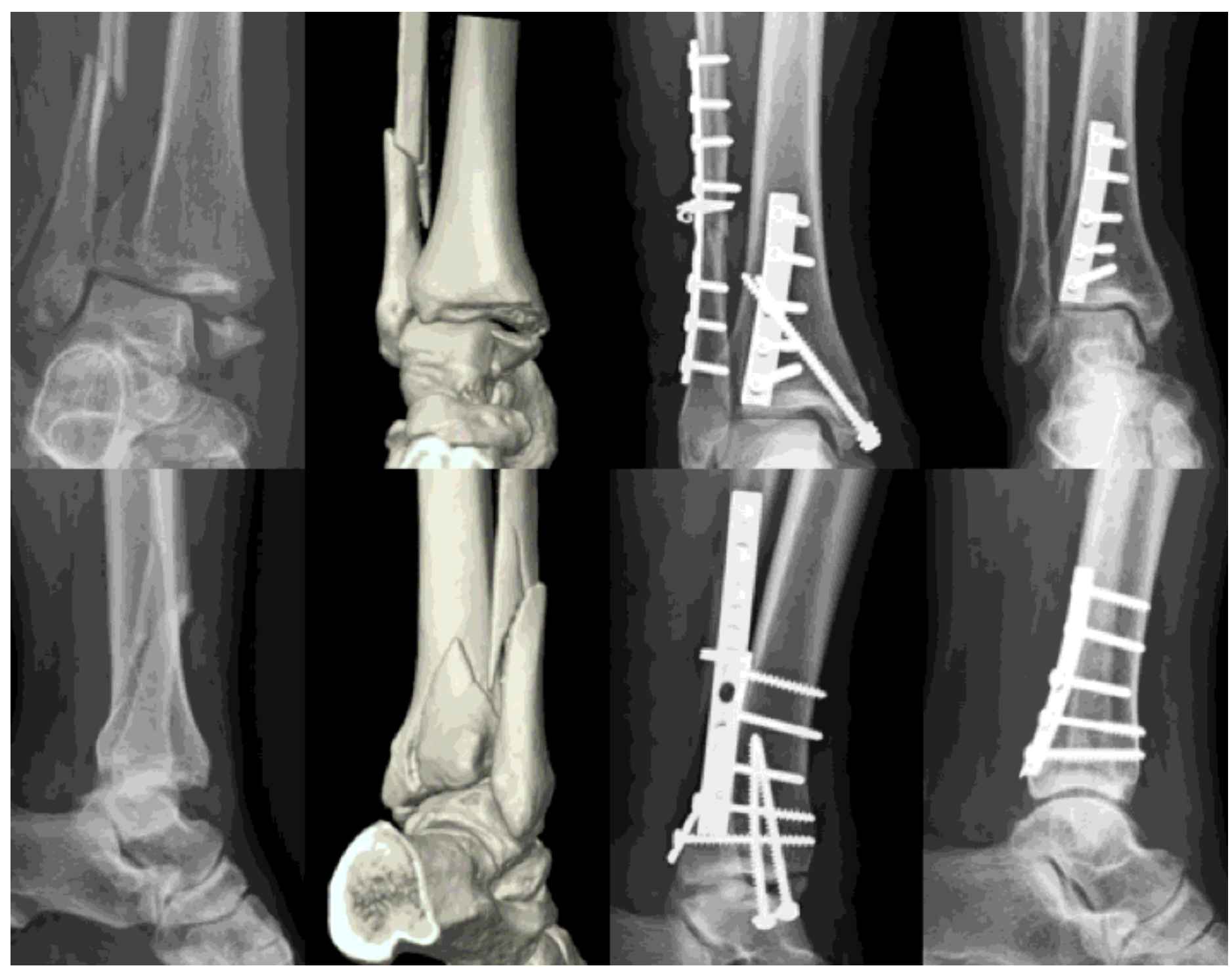

Figure 5

A 56-year-old woman sustained a right stage 4 pronation-external rotation ankle injury due to slide. Reconstructive computed tomography (CT) scans confirmed the lesions. Trimalleolar fractures were treated with screws and plates. Diastatic syndesmosis was not stabilized. The patient had a good ankle function at 4.3-year follow-up. 


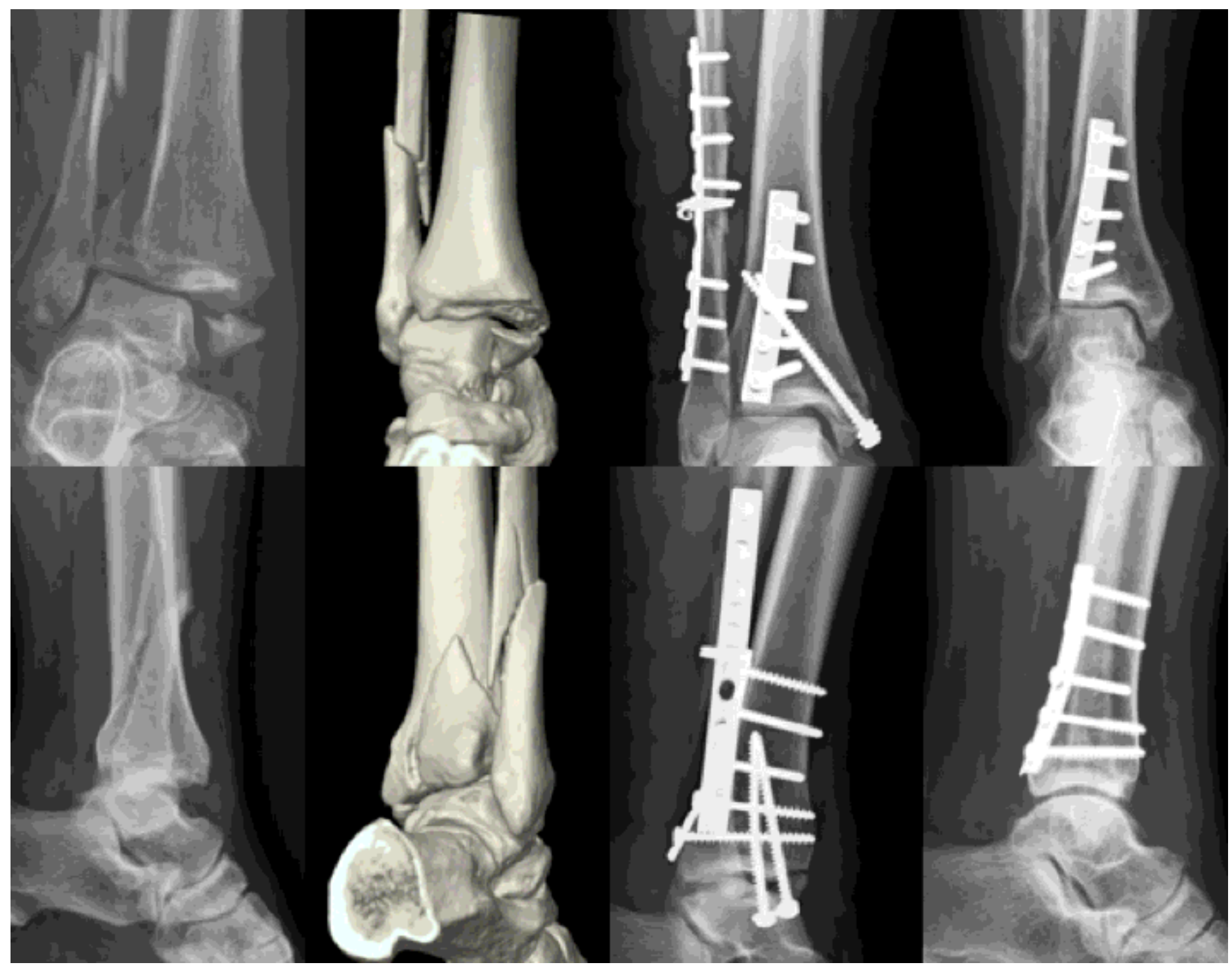

Figure 5

A 56-year-old woman sustained a right stage 4 pronation-external rotation ankle injury due to slide. Reconstructive computed tomography (CT) scans confirmed the lesions. Trimalleolar fractures were treated with screws and plates. Diastatic syndesmosis was not stabilized. The patient had a good ankle function at 4.3-year follow-up.

\section{Supplementary Files}

This is a list of supplementary files associated with this preprint. Click to download.

- PEadvdata.xIsx

- PEadvdata.xIsx

- PEadvdata.xlsx 